\title{
La gentrificación comercial y estigmatización a partir de la violencia. El caso del centro histórico de Ciudad Juárez, Chihuahua, 2009-2017
}

\section{Commercial gentrification and stigmatization from violence. The case of the historic center of Ciudad Juárez,} Chihuahua; 2009-2017

\section{Daniel Quezada Daniel*}

Recibido: 19 de enero de 2018

Aceptado: 06 de junio de 2018

\section{Resumen}

El fenómeno de violencia e inseguridad que experimentó Ciudad Juárez a partir de 2007 hizo que los diferentes ámbitos gubernamentales elaboraran estrategias de intervención y renovación urbana para disminuir los indicadores delictivos. El centro histórico fue donde se inició este proceso, debido que ahí se señalaban diversos comercios conocidos como giros negros (bares, cantinas, centros nocturnos y hoteles) que, a la luz del gobierno federal y local, fomentaban un ambiente de violencia. El objetivo del estudio fue analizar y describir este proceso de intervención urbana que tuvo como resultado la desaparición de estos comercios. La metodología utilizada fue de perfil mixto, sustentada en un análisis socioespacial en 2009 y 2017, a partir del Directorio Estadístico Nacional de Unidades Económicas (DENUE) e investigación en archivo de instituciones de comercio local. Los hallazgos exponen la desaparición de estos tipos de comercios de manera intensiva durante el periodo más álgido de violencia en Ciudad Juárez, en particular en la subzona turística. Los resultados muestran que la política de intervención se justificó en un contexto violento e inseguro, teniendo como base un diagnóstico en sus planes y programas que estigmatizaba las actividades comerciales, colectivos y lugares.

Palabras clave: centro histórico, estigmatización, gentrificación comercial, violencia.

\begin{abstract}
The phenomenon of violence and insecurity that Ciudad Juarez experienced from 2007 made the different governmental areas elaborate urban intervention and renewal strategies to reduce crime indicators. Since the historical center is where this process begins, because there were pointed out several shops known as black turns (bars, canteens, nightclubs and hotels) that were judged by the federal and local government as fostering an atmosphere of violence. The objective is to analyze and describe this process of urban intervention that resulted in the disappearance of these businesses. The methodology used is a mixed profile based on a socio-spatial analysis in 2009 and 2017, based on the National Statistical Directory of Economic Units (DENUE) and archival research of local trade institutions. The findings expose the disappearance of these types of businesses intensively during the height of violence in Ciudad Juarez, particularly in the tourist subzone. The results show that the intervention policy was justified in a violent and insecure context, based on a diagnosis in its plans and programs that stigmatized commercial activities, groups and places.

Keywords: Commercial gentrification, historic center, stigmatization, violence.
\end{abstract}

* Universidad Autónoma de Ciudad de Juárez, Ciudad Juárez, México.Contacto: danielqdaniel@gmail.com

Cómo citar: Quezada Daniel, D. (2018). La gentrificación comercial y estigmatización a partir de la violencia. El caso del centro histórico de Ciudad Juárez, Chihuahua, 2009-2017. Revista de Urbanismo, 38, 1-16. http://dx.doi.org/ 10.5354/0717$\underline{5051.2018 .48272}$ 


\section{Introducción}

En la actualidad los centros históricos viven un intenso proceso de intervención y renovación urbana impulsado por autoridades gubernamentales y sectores empresariales. Los efectos, que son estudiados desde diferentes ópticas en las ciencias sociales, comienzan con un cambio estético de los edificios, calles y plazas, para dar paso a la apropiación del suelo, implicando el desplazamiento de habitantes, colectivos y actividades comerciales tradicionales. Este fenómeno urbano ha creado una serie de clasificaciones muy particulares, que algunos estudiosos denominan aburguesamiento (Delgadillo, 2015) gourmetización o estudentificación (Carrión, 2005; Contreras, 2017), turistificación (Janoschka y Sequera, 2014), elitización (Smith, 1979), o bien, refiriéndose a la gentrificación como la situación en sí misma.

Este proceso de intervención y renovación en los centros de las ciudades mexicanas es observado con mayor auge desde 2007, cuando el gobierno federal impulsó una estrategia político-militar contra los grupos del narcotráfico justificando, a partir de este fenómeno, la implementación de los programas y proyectos de intervención para disminuir los actos violentos y delictivos que se estaban viviendo en los centros urbanos.

El sexenio de Felipe Calderón (2006-2012) inició con una estrategia de seguridad pública basada en combatir el control territorial que tenía el crimen organizado desde décadas anteriores. Sin embargo, los efectos fueron disputas más cruentas entre los propios grupos del narcotráfico y corporaciones de seguridad, teniendo como resultado altos indicadores de violencia en el país. Aún no está claro si efectivamente el narcotráfico suponía un problema de esa magnitud para implementar una amplia estrategia militar y policiaca, ni que la solución más adecuada fuera la confrontación violenta y directa (Vázquez y Espino, 2015). Incluso, sectores críticos y opositores, acusaron que la "guerra contra el narcotráfico" - como fue conocida dicha estrategiatenía un móvil eminentemente político, que se había sumado a un contexto de legitimidad del gobierno de Calderón (Chabat, 2010). También se afirmó que esta confrontación representaba los intereses de la clase media y alta, quienes habían votado en mayor medida por el candidato panista (Vázquez y Espino, 2015).
Con todo, es claro que uno de los resultados de esta estrategia fue el detrimento de la paz en México. Para el Institute for Economics and Peace (2016) el despliegue de cuerpos militares en actividades de seguridad ciudadana en los estados de Michoacán y Chihuahua provocó una escalada de violencia en todo el territorio nacional. Durante los cuatro años siguientes, el nivel de paz en México se deterioró en un $23 \%$, mientras que la tasa de homicidios casi se duplicó. En 2011 la tasa de homicidios para el Municipio de Juárez era de 148 por cada 100,000 habitantes, situando al estado de Chihuahua como la sexta entidad menos pacífica de México.

Por tanto, el aumento de violencia relacionada con la estrategia de combate a los grupos criminales impactó de manera progresiva en la seguridad pública de las ciudades, concentrando $70 \%$ de actos violentos en 85 municipios del país (Wolf y Morayta, 2011).

En este escenario, la mayor parte de esos hechos violentos fueron cometidos en asentamientos urbanos $y$ es ahí donde las políticas gubernamentales se enfocaron con mayor intensidad, emprendiendo operaciones militares y policiacas basadas en planes como Todos Somos Juárez, Reconstruyamos la Ciudad (TSJRC). Las críticas que acompañaban a este tipo de estrategias aparejadas del aumento de los indicadores de violencia, permitín generar un marco de interpretación que algunos académicos y especialistas enfatizaban -que la guerra contra el narcotráfico era más una acción de legitimización política por parte del presidente Felipe Calderón; que era una política de seguridad para combatir un problema deliberadamente magnificado (Vázquez y Espino, 2015)-, lo que se aunaba a la presión ejercida por el gobierno estadounidense al mexicano (Wolf y Morayta, 2011).

Por ello, la pregunta rectora de este trabajo plantea cómo el fenómeno de violencia e inseguridad experimentado a partir de 2007 justificó el proceso de renovación urbana del centro histórico de Ciudad Juárez, provocando la desaparición de comercios tradicionales conocidos como giros negros (bares, cantinas, centros nocturnos y hoteles), porque fomentaban ambientes peligrosos. Así, la hipótesis planteada señala que el fenómeno violento experimentado en esta ciudad fronteriza justificó un diagnóstico en sus programas y proyectos de intervención para impulsar el proceso de 
gentrificación del centro histórico, donde primeramente se estigmatizaron estos tipos de comercios, los cuales históricamente se habían establecido en esta parte de la ciudad desde inicios del siglo XX, para dar paso a nuevos establecimientos enfocados en consumidores de clase media y alta, quienes eran los que frecuentan la subzona turística del polígono de estudio. En el aspecto teórico, este trabajo tiene una mirada crítica del urbanismo a partir de la escuela francesa generada por el sociólogo Henry Lefebvre, pero también de los trabajos latinoamericanos de Carrión, Inzulza, Contreras, Delgadillo y Sabatini, quienes han tenido como objeto de estudio el centro histórico y su proceso de gentrificación.

En general, este artículo se integra de tres apartados: el primero, tiene la intención de plasmar la construcción del problema, sus orígenes y procesos actuales que vive la zona centro de Ciudad Juárez, Chihuahua; en segundo término, se abre la discusión teórico-conceptual de los centros histórico desde una mirada de la geografía y sociología crítica, sostenida en el análisis del fenómeno de violencia e inseguridad que surge de modo transversal para justificar el proceso de gentrificación, así como también se integra la propuesta metodológica basada en trabajo de archivo en la Dirección de Comercio en Ciudad Juárez, recorridos de campo, y análisis socioespacial del Directorio Nacional de Unidades Económicas (DENUE); por último, en el tercer apartado se exponen los resultados y el análisis socioespacial de las actividades comerciales para 2009 y 2017, señalando aspectos relevantes como las estrategias utilizadas por las autoridades para desaparecer comercios del centro histórico (bares, cantinas, centros nocturnos y hoteles), sobreviviendo solo aquellos negocios del mismo perfil, pero destinados a sectores sociales de alto consumo.

\section{Gentrificación, estigma y desaparición de comercios}

En la actualidad se gesta e intensifica un proceso de gentrificación sobre los centros históricos. El suelo e inmuebles públicos, plazas, parques, reservas naturales, vialidades, servicios sociales y áreas recreativas se van entregando a las empresas productivas, comerciales $y$ servicios, desplazando o, en situaciones extremas, haciendo desaparecer colectivos y comercios tradicionales que habían hecho del núcleo central su punto nodal de vida.
En Latinoamérica existe una amplia discusión académica que en torno a las diversas configuraciones urbanas surgidas de la gentrificación, resaltando aquellos cuestionamientos de los procesos de desplazamiento voluntario, coaccionado o forzado de la población y que se deriva de las políticas urbanas (Delgadillo, 2015); de las desigualdades en torno a la renta del suelo y el desplazamientos excluyentes (López-Morales, Klet y Corvalán, 2014); o bien, de una gentrificación que surge como resultado de los desastres naturales (InzulzaContardo y Díaz, 2016); sin olvidar aquellos análisis que ponen en el centro de la discusión al sector inmobiliario que gentrifica y, a su vez, segrega a otros sectores de la población (Sabatini, Rasse, Cáceres, Robles y Trebilcock, 2017).

Sin embargo, en esa diversidad de aristas se puede resaltar que el nexo de la gentrificación representa la confluencia de diferentes fuerzas hacia la producción paulatina de una mayor homogeneidad y lugares privados, cada vez más integrados a la lógica del mercado. Por tanto, para entender qué es la gentrificación se tienen que reconocer sus componentes. En primer lugar, se trata de un fenómeno urbano que apareció en las lecturas académicas a mediados de 1960 gracias a Ruth Glass, quien acuñó dicha categoría para denominar los cambios ocurridos en determinados barrios londinenses y que presentaban un creciente deterioro ante la llegada de nuevos residentes de poder adquisitivo medio-alto. A partir de ahí, se desarrollaron diversas lecturas de este fenómeno social, ya fuera desde la mirada sociológica, como de la económica, política o cultural.

Ahora bien, debe concebirse en la visión económica una dicotomía clara: los procesos de gentrificación se explican como un fenómeno que abarca la oferta y la demanda, las dimensiones de la producción y del consumo. De la misma forma que los consumidores (co)producen la gentrificación y el espacio urbano; los productores también consumen (Delgadillo, 2016) generando ciclos que resultan en el desplazamiento de la población o en la desaparición de comercios tradicionales. Es decir, desde la perspectiva de la demanda la gentrificación se encuentra basada en los cambios de los patrones de consumo, siendo la gente joven, generalmente profesional y de clase media, quienes van transformando su estilo de vida. El perfil demográfico de este grupo apunta a tener menos hijos, aplazamientos de 
matrimonios y un rápido aumento en la tasa de divorcios. Por tanto, los compradores de vivienda tienen características definidas: jóvenes, altos niveles de consumo y decisiones guiadas por usos de suelo en la ciudad (Ley, 1978, p. 11 citado por Smith, 1979). A partir de ello, López-Morales et al. (2014) aducen que los cambios culturales y sociodemográficos reposicionan la demanda residencial por el suelo urbano central, generando nuevas inversiones inmobiliarias.

El otro sentido de la dicotomía económica anteriormente mencionada, es en los estudios de gentrificación sustentados en la teoría neoclásica donde la suburbanización refleja una preferencia por el espacio y capacidad de pago del consumidor (oferta). De manera similar, la gentrificación se explica como resultado de una alteración de las preferencias generadas por los inversionistas y el capital. Al respecto, López-Morales et al. (2014) clasifican esta posición como una visión estructural (oferta/inversión), donde los gentrificadores no necesariamente son los grupos sociales que llegan a vivir a un barrio, sino la clase social que genera producción inmobiliaria en mayor escala y que estructura la oferta y el desarrollo del barrio. Desde esta perspectiva, el patrimonio histórico, cultural y los valores del entorno urbano, así como el significado de centralidad y las oportunidades de inversión van impulsando la llegada de nuevos residentes con mayores niveles de ingreso, que más allá de aprovechar las ventajas que ofrece el centro histórico, buscan nuevas necesidades de interacción y de representación social (Gastaldi y Camerin, 2015).

La crítica realizada a estas dos líneas radica en que, si la visión del consumo (demanda) y la disposición por parte de los inversionistas (oferta) realmente explican la gentrificación, esto equivaldría a suponer que las preferencias individuales cambian no solo de manera nacional, sino también internacionalmente. A pesar de estas discusiones, sigue siendo la variable económica la que tiene mayor relación con la elitización urbana, porque es el producto esperado de la libre operación del mercado del suelo y de la vivienda.

En otras palabras, los procesos de gentrificación se apoyan en el aprovechamiento de las denominadas rentas urbanas, generadas por la diferencia entre el precio del suelo actual y el precio del suelo futuro, producto de un cambio en el uso del mismo, del aumento del límite de construcción y por mejoras en los inmuebles o por obras públicas urbanísticas (Contreras, 2017).

A partir de esta diferenciación y discusión en torno a la gentrificación desde una perspectiva económica, van surgiendo tres actores relevantes que tienen incidencia en este ciclo urbano para observar sus regularidades de acción: 1) el sector privado-inmobiliario que mira los espacios intervenidos como un punto de (re)inversión de su capital; 2) el Estado como un actor que promueve, invierte y genera las condiciones para que se realice el proceso; y 3) la población, colectivos y comercios tradicionales que sufren las consecuencias por medio de la desaparición o, en el mejor de los casos, el desplazamiento hacia otras partes menos favorecidas de la ciudad.

Hasta este momento el análisis permite dilucidar algunas regularidades: las autoridades locales revitalizan áreas en declive o promueven determinadas zonas urbanas; los desarrolladores, por su parte, emplean la investigación de mercado, la focalización, y el diseño, entre otras técnicas, para concebir un lugar mediado por el consumo; la creación de marcas y las relaciones públicas elaboran un discurso positivo para legitimar el nuevo lugar y; por último, con el tiempo, el lugar obtiene legitimidad (Castilhos, 2015).

Es dable suponer, entonces, que el sector inmobiliario tiene gran interés en que estos procesos se concreticen, ya que obtiene los mayores beneficios. La inversión en el suelo es parte de la acumulación del capital, porque es un medio de producción en el circuito de la economía actual. Los cambios globales son particularmente notables para entender cómo las ciudades, los suburbios y las regiones son afectados por la economía en los últimos años y, sobre todo, la inversión en bienes raíces ha sido esencial en la reestructuración del espacio (Gottdiener, Hutchison \& Ryan, 2014). Al respecto, se debe entender la lógica del sector inmobiliario y financiero junto con sus pautas de inversión no de manera exclusiva hacia el desplazamiento de la vivienda de interés social construida en megaconjuntos o los megaproyectos inmobiliarios mixtos destinados a las actividades empresariales y vivienda de sectores de altos ingresos (Pradilla, 2013). También las inversiones de estos sectores miran hacia el centro de las ciudades como espacios redituables, donde van (re)construyendo las áreas centrales con sus productos 
emblemáticos: centros comerciales, torres de usos mixtos y conjuntos cerrados.

Si bien, Ciccolella (2010) afirma que las clases medias se van apropiando cada vez de los principales lugares del suburbio al igual que retornando al centro, en ocasiones no siguen dicho patrón: el uso del suelo del centro no tiende hacia lo habitacional, sino a servicios de alto valor, llegando a adueñarse el capital de los mejores lugares para el consumo de bienes culturales y turísticas de los centros históricos.

En definitiva, los centros no solo tienen que ser analizados desde los atributos históricos que tienen sus construcciones, sino también a partir de las características que tienen los actores que inciden sobre los proyectos y las directrices de intervención urbana que provienen del ámbito internacional, expresadas en entidades como la Organización de las Naciones Unidas para la Educación, la Ciencia y la Cultura (Unesco), que es la encargada de velar por la protección del patrimonio mundial y en el ámbito nacional o local están patronatos, asociaciones, los pequeños comerciantes y habitantes de esos lugares (Unesco, 2013).

Todos estos actores tienen en común un espacio de consumo que coincide con los lugares históricos de la acumulación de capital, transformando los espacios de significado histórico en espacios del mercado: un espacio concreto y cuantificado por dimensión, inversión y flujos (Lefebvre, 1974).

Es ahí donde el centro histórico se posiciona como un espacio endeble y codiciado para ser intervenido por parte de los inversionistas en conjunto con los gobiernos. Por ello, en Latinoamérica se vive este fenómeno de intervención urbana, que revaloriza la ciudad construida, y dentro de ella, con un grado aún mayor, los dos tipos de centralidades: la urbana y la histórica, en un contexto de internacionalización. La primera, urbana, se refiere al constructo de funcionalidad y flujos que parten del centro hacia fuera o periferia de la ciudad. La segunda, histórica, es el espacio por excelencia de encuentro, tanto por su condición de centralidad -que hace que sea un punto focal de la ciudad-, como por la dimensión temporal que le permite adquirir un valor de historia. Un agente preponderante en este proceso es el capital especulativo financiero e inmobiliario que se enfoca en comprar los mejores lugares de las ciudades, desplazando hacia las periferias a las clases bajas y, en casos extremos, a los sectores medios (Carrión, 2005).

En el contexto de estos cambios que está sufriendo la ciudad en América Latina, la centralidad histórica debe readecuarse a las nuevas funciones, recurriendo a la promoción y construcción de los grandes proyectos urbanos (Carrión, 2005). Aunque para entender las centralidades y en específico las históricas, es necesario, primeramente, definirlas sobre la base de diversas características, desde lo histórico, pasando por lo simbólico y lo económico.

Siguiendo a Carrión (2014) se tienen tres momentos para poder categorizar un centro histórico. En primera instancia, como una definición monumentalarquitectónica (una iglesia o una plaza); en segundo lugar, el monumento en relación con la ciudad; y, en tercer término, contextualizando todo el conjunto monumental, es decir, la suma de los monumentos. De ahí que es difícil entender una centralidad histórica sin su referencia monumental, esto es, sumado a las relaciones sociales y nodales que genera.

De esta forma, la centralidad histórica debe entenderse más por su sentido de cómo fue fundada, la función que realiza y la temática que le ha sido concedida en la globalización. El primer destino es fundacional, o sea, tiene una noción de lo antiguo religioso y político, un centro considerado con esencia pública $y$, por ende, abierta. La segunda ocupación es funcional, es decir, la centralidad histórica se concibe en relación con su centralidad respecto de la ciudad y, por lo regular, contiene actividades comerciales y financieras, siendo un espacio pluricentral, privado y cerrado. La tercera función, temática, comprende las relaciones interurbanas, nodales, público-privada y de "no lugares", en donde se trata de un ámbito de actividades del nivel terciario superior (Carrión, 2017).

En la actualidad, los centros históricos son reflejo de tensiones marcadas entre los sectores populares que viven en esos espacios y la gentrificación del espacio urbano, debido a la llegada servicios y comercios de alto valor. Esto implica un proceso de expulsión/desaparición de la población, porque cambia el uso del suelo a través de esas actividades que pagan altos costos de esta localización y, por consiguiente, entra en conflicto la 
pobreza que tiene la población frente a un capitalismo de perfil turístico.

Es relevante enmarcar que el conflicto surge en el momento en que las autoridades encargadas de la intervención urbana señalan los espacios como sitios inseguros y violentos. En un contexto teórico, Wacquant, Slater y Borges Pereira (2014) nombran a este proceso como estigmatización territorial. El término estigma hace referencia a un atributo que desacredita al individuo o al colectivo, por cuestiones raciales y étnicas, lo cual confluye en ser observados como inferiores, peligrosos y repulsivos. En este caso se retoma el estigma como una categoría explicativa, pero añadiendo la cuestión territorial.

Por tanto, la estigmatización territorial no es una condición estática o un proceso neutral, sino una forma significativa y perjudicial de acción mediante la representación colectiva centrada en un lugar determinado Wacquant et al. (2014). Desde esta óptica, el estigma generado como un atributo negativo se complementa con una perspectiva espacial, entonces en el transcurso del desarrollo se van integrando otros atributos, no solamente raciales y étnicos, sino también otros relacionados con la pobreza, las viviendas degradadas, las moralidades impuestas y la delincuencia.

Con el tiempo, los actores sociales (los medios de información, políticos, académicos e instituciones gubernamentales) llegaron a democratizar el término, ya que actualmente en cada ciudad existe por lo menos un territorio estigmatizado: la calle del comercio sexual, la de comercio informal e ilegal, la zona de tugurios o el barrio de los pobres. Desde el ámbito local son conocidos y señalados con esos atributos que recaen en lo obscuro, prohibido y moralmente repulsivos. Se genera así una diversidad de emociones negativas y reacciones severas correctivas, impulsadas ya sea por miedo o el rechazo, dando paso a discursos urbanos que señalan la necesidad de rescatar esos espacios, como si hubieran sido absorbidos por la perdición en el transcurso de la historia.

Este trabajo incluye los comercios como los bares, cantinas, centros nocturnos y hoteles, como reflejo de ese estigma territorial, que es donde se reúnen las clases más desfavorecidas y son considerados espacios de perdición y lúgubres.
En palabras de August (2014), los discursos de aislamiento, desorganización y peligro que tienen esos territorios estigmatizados sirven para justificar la gentrificación, puesto que el desprestigio territorial revela cómo las instituciones encargadas de las políticas urbanas elaboran y vinculan el concepto de estigma con el territorio y los elementos que contiene, para posteriormente justificar la demolición, expropiación o clausura de comercios, sin observar los diversos factores que han llevado al establecimiento de esos lugares.

Por tanto, se establece que la gentrificación no corresponde únicamente al desplazamiento de sectores sociales de ingresos bajos, sino que también articula un proceso transformador de actividades comerciales que desaparecen o se desplazan hacia otra parte de la ciudad. Así, se implementan modelos comerciales que ofrecen satisfacciones que demandan los nuevos pobladores, pero con la característica de ser especiales y únicos, atraídos por la gourmetización o fashion mall, que los nuevos negocios les pueden ofrecer.

La descripción de todo el proceso gentrificador en los centros históricos vislumbra elementos que se van integrando. El gobierno interviene el territorio bajo una premisa de renovación para rescatar esos espacios urbanos, por consiguiente, tiene un efecto multiplicador: los precios del suelo son bajos debido al deterioro sufrido $\mathrm{y}$, en el momento que comienzan las renovaciones, el precio sube. Son los nuevos habitantes de estratos económicos más altos quienes tendrán mayor capacidad de consumo y, por ende, desplazarán a las antiguas clases sociales de ingresos inferiores, que no pueden hacer frente al aumento del costo de vida y los precios de bienes inmuebles.

Es relevante señalar que, según Cordero y Salinas (2017), la transformación comercial corresponde a un esquema de competitividad urbana, donde las ciudades se enfocan en impulsar territorios renovados que sean la punta de lanza del sector turístico, financiero e inmobiliario, siendo en esta reestructuración reemplazados los comercios por otros de mayor valor, más competitivos y con una conversión residencial rentable típica de la era postindustrial (Ferm, 2016). De esta manera la percepción de la gentrificación comercial puede ser observada en sentido dicotómico: por un lado puede mirarse como oportunidad y, por otro, como un 
problema. La primera promueve trabajos asequibles y se confía en el desarrollo dirigido por el sector privado para asegurar los beneficios sociales. La segunda, refleja la preocupación que impulsa el inversionista y planificador, quienes relegan las necesidades de los antiguos residentes.

En conclusión, el proceso de gentrificación está en correspondencia con el actual sistema económico, el cual no solamente invierte en zonas de la periferia de la ciudad, sino que también regresa a la centralidad histórica, a través de las asociaciones público-privadas (APP) que tienen como secuela el desplazamiento y desaparición de actividades económicas, además de la expulsión de colectivos, lo que deja esa zona de la ciudad a merced de las intervenciones de mercado. En síntesis, la gentrificación no es el producto de una política urbana, sino que más bien es visualizada como una única y exclusiva política por parte de las autoridades para aquellos territorios estigmatizados como peligrosos, abandonados y deteriorados.

\section{Enfoque metodológico}

La metodología utilizada es de perfil mixto, sustentada en un análisis socioespacial para 2009 y 2017. Consistió en georreferenciar los establecimientos señalados como giros negros (bares, cantinas, hoteles y centros nocturnos). Las fuentes primarias de información fueron el registro mercantil municipal de 2009 y el Directorio Estadístico Nacional de Unidades Económicas (DENUE) para 2017. Además se realizaron recorridos de observación no participante en toda la delimitación del centro histórico, con el objetivo de registrar el tipo de comercios que desapareció en las zonas intervenidas por el Instituto Municipal de Investigación y Planeación ,IMIP, y aquellos que lograron mantenerse.

De esta forma, el proceso metodológico contempló cuatro etapas. En la primera se definieron antecedentes, problematización del fenómeno, supuestos y objetivos de investigación, además de delimitar el área de estudio en función del Plan Maestro de Desarrollo Urbano del Centro Histórico de Ciudad Juárez, PMDUCHJ. En la segunda etapa se retomó el análisis de las categorías explicativas (la gentrificación de los centros históricos apoyada en la estigmatización). En la tercera fase se plantearon las dimensiones de observación: la histórica, la económica, la social y la política urbana. La cuarta instancia consistió en organizar la información y fuentes para continuar con su análisis, con el objetivo de dar respuesta al proceso de desaparición de los comercios estigmatizados por las autoridades, además de presentar los hallazgos y su relación conforme a su significado para el estudio.

De igual manera, la cuestión espacial se basó en las políticas de intervención urbana aplicadas por el gobierno municipal de Juárez en el centro histórico, siendo el universo de estudio y unidades de análisis estas cuatro actividades comerciales señaladas por las autoridades por fomentar actos violentos e inseguros en esa parte de la ciudad, esto es, bares, cantinas, hoteles y centros nocturnos.

\section{Los efectos de la intervención en el centro histórico de Ciudad Juárez}

En 2008 y 2009, Ciudad Juárez fue considerada la ciudad más violenta del mundo (Seguridad, Justicia y Paz, 2016). Partiendo del caso paradigmático de esta ciudad fronteriza del norte mexicano, que es un referente del fenómeno de violencia en el ámbito nacional e internacional, se pusieron en marcha diversas políticas de seguridad policial y social por parte del gobierno federal, como la estrategia antes mencionada (TSJRC), que fue la base para la elaboración de programas parciales y puntuales, como el Plan Maestro de Desarrollo Urbano del Centro Histórico de Ciudad Juárez (PMDUCHJ), elaborado por el Instituto Municipal de Investigación y Planeación (IMIP), teniendo como trasfondo solucionar la situación de violencia e inseguridad en el primer cuadro de la ciudad fronteriza, a través del mejoramiento e intervención de los espacios urbanos considerados por las autoridades como peligrosos.

Estos planes estuvieron basados y justificados a partir de hechos violentos que tuvieron como escenario lugares del centro histórico de Ciudad Juárez. Por citar algunos ejemplos, y que resonaron en el ámbito nacional, fue el proceso llamado Juicio del Siglo, siendo el punto de disputa un inmueble de tres niveles, localizado en el primer cuadro del centro histórico y conocido como el Hotel Verde o Salón Verde, donde fueron localizadas varias mujeres con registro de desaparición en esa zona entre 2008 y 2010, y que eran obligadas a ejercer el comercio sexual. En la actualidad este inmueble, que 
guarda la historia de numerosas mujeres, permanece cerrado y en una situación jurídica ambigua (Castañón y Carmona, 2015).

Un segundo caso de gran impacto mediático sucedió en 2010, cuando un vehículo cargado con explosivos fue estacionado por integrantes del crimen organizado en las calles de 16 de septiembre y Bolivia, muy cerca del centro histórico. Un hecho que es recordado por el gremio de los médicos debido que uno de sus miembros falleció en ese lugar, prestando ayuda como voluntario (González, 2010). El hecho tuvo resonancia mediática debido que fue catalogado por las autoridades como un acto terrorista, ya que el explosivo fue activado de manera remota.

En ambas situaciones - que tuvieron mayor atención por parte de los medios de comunicación nacional e internacional一, pusieron en el escenario la discusión al centro histórico como un territorio endeble y generador de violencia e inseguridad en toda la ciudad, llevando a declarar al entonces presidente municipal Héctor Murguía Lardizábal, "la necesidad de intervenir el centro para darle un nuevo rostro, ya que no veía otra manera de erradicar la violencia y la podredumbre que se concentraba y emanaba casi en su totalidad en el centro histórico, más que tirando y volviendo a construir" (Chaparro, 2013).

Aunque, años después, los informes oficiales señalaban una disminución de actos violentos en Ciudad Juárez, no dejó de ser un problema prioritario para los habitantes fronterizos, donde la inseguridad y violencia se ubicó en el primer lugar con 19,4\%, por encima de la corrupción (15,9 \%), pobreza $(11,3 \%)$, drogadicción $(7,7 \%)$, calles y pavimentación $(4,1 \%)$ o infraestructura (17\%). Sin embargo, al analizar si vivir en esta ciudad fronteriza podría ser considerado como seguro o inseguro un $40,6 \%$ de la población creía que era muy seguro o algo seguro, frente al 38,9\% de los habitantes que consideró a Ciudad Juárez como algo inseguro o muy inseguro (Plan Estratégico de Juárez, 2016).

En resumen, es a partir de 2007 que se implementa una estrategia militar y policiaca replicada en la mayoría de las ciudades de México, con el argumento de combatir el crimen organizado, sin embargo, todo este fenómeno ha elevado los indicadores de violencia e inseguridad. Más allá del dato estadístico, que no deja de ser relevante para los estudios, nos acerca a un panorama de la realidad que viven las ciudades, donde los municipios urbanos son los que tienen mayor incidencia de este fenómeno.

Asimismo, esta estrategia fue el parteaguas para impulsar programas y planes que tuvieran como esencia mejorar las condiciones de seguridad de la población, teniendo como antecedentes y justificación los actos violentos perpetrados en los principales centros urbanos, con lo cual se fueron estigmatizando los territorios como parte del problema. Parafraseando a Wacquant (2006), un problema político y económico se transforma, primeramente, en el discurso y luego en los hechos, en un problema espacial de criminalidad, donde los grupos y colectivos sociales son punto de referencia para su desplazamiento, aunque las actividades comerciales como bares, cantinas, centros nocturnos y hoteles donde se ejerce el comercio sexual, se convirtieron para las autoridades en un objetivo esencial para su desaparición del centro histórico. Es decir, todos estos actos violentos y las posteriores declaraciones realizadas por los diversos sectores sociales y gubernamentales han servido como respaldo y justificación para aplicar programas y proyectos de intervención urbana como fue el caso del PMDUCHJ.

\section{Desplazamiento de actividades comerciales}

En una de sus últimas entrevistas que concedió el escritor juarense Víctor Bartoli en 2009, afirmaba que en Ciudad Juárez siempre había existido la desaparición de mujeres en el centro de la ciudad y, por ende, ya era considerada una urbe peligrosa. No era un fenómeno actual, sino por el contrario, sus inicios pueden remontarse a 1940 con la llegada del fuerte Fort Bliss a El Paso, Texas, situada a pocos kilómetros de la frontera norte de México (Jamieson, 1993) donde Juárez se convirtió en la gran proveedora de placer sexual para los soldados estadounidenses, quienes llegaban a cometer violaciones y asesinatos de mujeres. A pesar de esto, el comercio sexual se asentó aún más con el proceso migratorio de los braceros provenientes de todas las regiones del país, donde algunos terminaron por establecerse de manera formal en esta ciudad mexicana.

Así, no es casualidad que, en la década de 1970 sea caracterizada la zona centro junto a sus avenidas principales -Juárez, Vicente Guerrero, 16 de septiembre e Ignacio Mariscal-, como espacios donde se 
concentraba la mayoría de los centros nocturnos, salones de baile, cantinas, hoteles y tugurios de toda la ciudad.

Por ello, al hablar de Ciudad Juárez, es imprescindible mencionar la vida nocturna del centro y la generación de otras actividades que se desarrollaron alrededor, como el servicio de hospedaje, vinaterías, transporte y pequeño comercio, que en letras de Peña y Sandoval (2010) las noches eran largas, uniéndose en vigilia con las madrugadas, una ciudad que no se agotaba, simplemente una avasalladora vida nocturna; creación artística de vanguardia, donde el reconocimiento y el placer inmediato del público se satisfacía, viendo el espectáculo de los cabarets en vivo.

De esa manera, Ciudad Juárez y en específico la zona centro, fueron consideradas en su momento como Las Vegas de México, aunque debido a diversas circunstancias esta ciudad fue experimentado otros adjetivos de acento moralizante como Ciudad del pecado, Capital del vicio, Pozo del mundo y en los últimos años como J-war-ez (Padilla, Andradey Salas, 2014). Esta última referencia, hace alusión a un estado de guerra (war) que corresponde a la estrategia policiaco-militar que inició el gobierno panista de Felipe Calderón a partir de 2006, como una política de respaldo popular y que demostrara el poder del Estado ante quienes hubiesen pensado que el proceso electoral había dejado un presidente débil (Lara y Enrique, 2011).

A partir de 2007, Ciudad Juárez vivió una escalada de violencia exponencial. La mayoría de los asesinatos se relacionaban con la disputa por la frontera entre dos carteles de la droga, el de Sinaloa y el de Juárez, que a través de pandillas como los Mexicles, Aztecas o Artistas Asesinos peleaban cada colonia o esquina de esta ciudad fronteriza.

La estrategia Todos Somos Juárez y Reconstruyamos la Ciudad, fueron impulsados desde el ámbito federal y con participación del gobierno estatal y local. El planteamiento se sustentaba en 160 acciones en los temas de economía, empleo, salud, educación y desarrollo social. El objetivo esencial era disminuir los niveles de violencia que se habían estado presentando desde 2007. Los compromisos que hacen referencia a las intervenciones espaciales es el compromiso 7, que refiere a "Revisar los centros de esparcimiento nocturno para garantizar su funcionamiento en cumplimiento de la reglamentación aplicable y aislarlos de la lógica delictiva" y el compromiso 144, donde se constituye el Patronato Pro Espacios Públicos de Juárez, con la participación del sector empresarial para garantizar la viabilidad financiera y operativa de los espacios públicos, entre ellos el centro histórico de Ciudad Juárez. Por último, el compromiso 145 hace referencia a la movilidad integral: elaborado por el Instituto Municipal de Investigación y Planeación (IMIP) este compromiso define las estrategias y acciones prioritarias de planeación y movilidad urbana (Gobierno Federal, 2010). A partir de esta estrategia federal se impulsa el Plan Maestro de Desarrollo Urbano del Centro Histórico de Ciudad Juárez con el objetivo de intervenir la zona centro, teniendo como características: su esencia de centralidad, la cercanía estratégica con el puente fronterizo internacional, ser el principal nodo de transporte, atracción turística $y$, sobre todo, ser el perímetro donde se había registrado el mayor número de desaparición de mujeres.

En suma, el interés privado por este sector radicaba en su localización privilegiada y el potencial económico que proyectaba, aunque el principal problema era el tipo de comercio y servicios, los que no eran acordes con el proyecto de renovación. Es por ello que el desplazamiento o desaparición de colectivos y grupos sociales, así como el tipo de actividades que fomentaban violencia e inseguridad en la zona se realizó mediante dos estrategias: la primera corresponde a la negociación y acuerdos con el sector empresarial de la ciudad, o bien, con los dueños de comercios y predios de la zona por medio de mesas y foros de discusión. De las propuestas que surgieron de estos espacios de diálogo resaltan los incentivos fiscales, apoyos y fideicomisos. Por tanto, se comenzaron a gestionar los alicientes, así como también se procedió a certificar las restauraciones para solicitar los subsidios al finalizar las obras, o bien coadyuvar a la obtención de los créditos para apoyar las actividades comerciales y de servicios (IMIP, 2013). Los anteriores apoyos correspondieron a inversión pública, inversión mixta, de crédito y de financiamiento 
nacionales e internacionales ${ }^{1}$, los que estuvieron destinados a generar alta rentabilidad y crear plusvalías directas a los inmuebles. En algunas situaciones, las inversiones por parte del gobierno fueron destinadas a asuntos privados (fondo perdido). Se trató, entonces, de un desplazamiento pasivo y no forzado, basado en el acuerdo económico.

En cambio, la estrategia de desplazamiento y desaparición de colectivos y grupos sociales de la zona fue diferente. En entrevistas realizadas a dueños de comercios de bares y cantinas, es constante la referencia al incremento del hostigamiento por parte de las autoridades, llegando las personas a ser remitidas en instalaciones policiacas por cometer supuestas faltas administrativas. $^{2}$

El cierre y la destrucción de decenas de locales y fincas, especialmente de viviendas y hoteles de paso, afectó directamente a las trabajadoras sexuales que laboraban dentro de estos negocios, pues no se reubicó a ninguno de ellos en otra zona ni sus propietarios fueron indemnizados por sus terrenos (Rubio, 2013).

Bajo ese marco del proceso de intervención urbana y las estrategias realizadas por las autoridades, la metodología que sustentó este estudio fue georreferenciar aquellas actividades económicas indicadas como giros negros, distribuidos en la delimitación propuesta por el IMIP. El perímetro de estudio comprendió seis subzonas, conforme al PMDUCH: comercio, turística, administrativa, equipamiento, servicios, vivienda y abastecimiento.

Ahora bien, en 2009 de acuerdo con los datos obtenidos, se tenía un registro de 126 establecimientos catalogados como bares, cantinas y centros nocturnos (Figura 1). Para su clasificación como giros negros, las autoridades señalaron el aspecto más relevante: debía existir el consumo de bebidas alcohólicas. En cuestiones de uso de suelo estos no deberían estar en zonas habitacionales o cerca de centros educativos, pero sí fueron permitidos en la subzona turística, comercial y de servicios.

De esta manera, la mayoría de los establecimientos se localizaba sobre la avenida Juárez, que es el principal acceso de circulación peatonal y vehicular a Estados Unidos. En paralelo a esta arteria vial se encontraba la calle Mariscal, donde también se ubicaban negocios de expendio de bebidas alcohólicas. La diferencia entre ambas calles no radicaba en su dimensión, características físicas de sus establecimientos o en su accesibilidad, sino negocios.

\footnotetext{
${ }^{1}$ La inversión pública realizada de 2007 a 2017 fue de $\$ 134,486,190.48$ millones de pesos mexicanos. Para 2007 fue de $\$ 20,749,839.60$, y para $2017, \$ 31,844,395.27$. Es decir, la inversión pública en infraestructura, mobiliario e imagen del centro ha sido constante durante el periodo de violencia en Ciudad Juárez.
} 

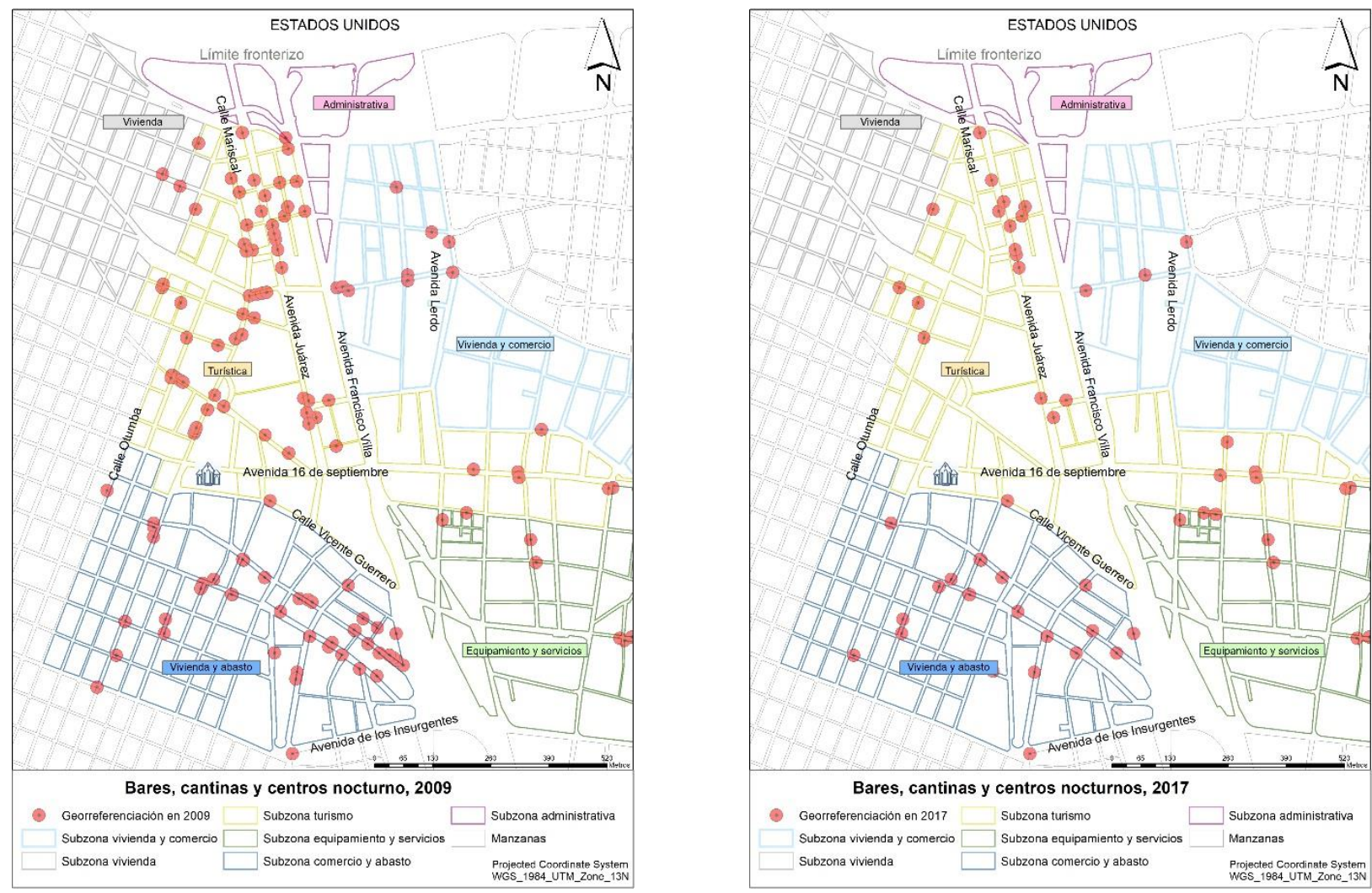

Figura 1. Bares, cantinas y centros nocturnos 2009-2017.

Fuente: Elaboración propia con base en DENUE y archivo Dirección Comercio Municipal de Ciudad Juárez.

La avenida Juárez siempre fue distinguida por encontrarse en ella bares como el Club Kentucky, considerado como un lugar histórico, porque en algún momento fue visitado por Elizabeth Taylor, Jim Morrison, John Wayne, Steve McQueen y Marilyn Monroe; y por su supuesta invención de la bebida Margarita. Sobre esta misma avenida se localiza el centro nocturno Noa-Noa, que impulsaría la vida artística del ícono de la música popular mexicana Juan Gabriel.

En contraste, la calle Mariscal fue un referente para el consumo de bebidas alcohólicas frecuentado por trabajadores, avecindados, migrantes y jornaleros, quienes veían en esta calle un espacio de diversión y disfrute, ya que no existían otros lugares en la ciudad que les ofrecieran una variedad de establecimientos con precios accesibles.

Alrededor de ambas avenidas se fueron desarrollando otros tipos de negocios, en especial aquellos dirigidos al hospedaje, ya que era un servicio muy utilizado por los migrantes, porque les permitía pernoctar un tiempo por una módica cantidad de dinero, aunque antes de la intervención del centro era también común observar a personas que ejercían el comercio sexual afuera de estos edificios.

A partir del PMDUCH, la distribución y número de estos establecimientos cambiaría de manera drástica, llegando a sobrevivir pocos comercios sobre la avenida Juárez y algunos en la parte sur del perímetro.

En 2009 se tenía 36 hoteles señalados como referentes del comercio sexual; y de bares, cantinas y centros nocturnos se contemplaba 126 establecimientos (Figura 1). Para 2017, en tanto, el número hoteles llegó a 27, sobreviviendo 55 espacios dedicados a bares, cantinas y centros nocturnos (Figura 2). 

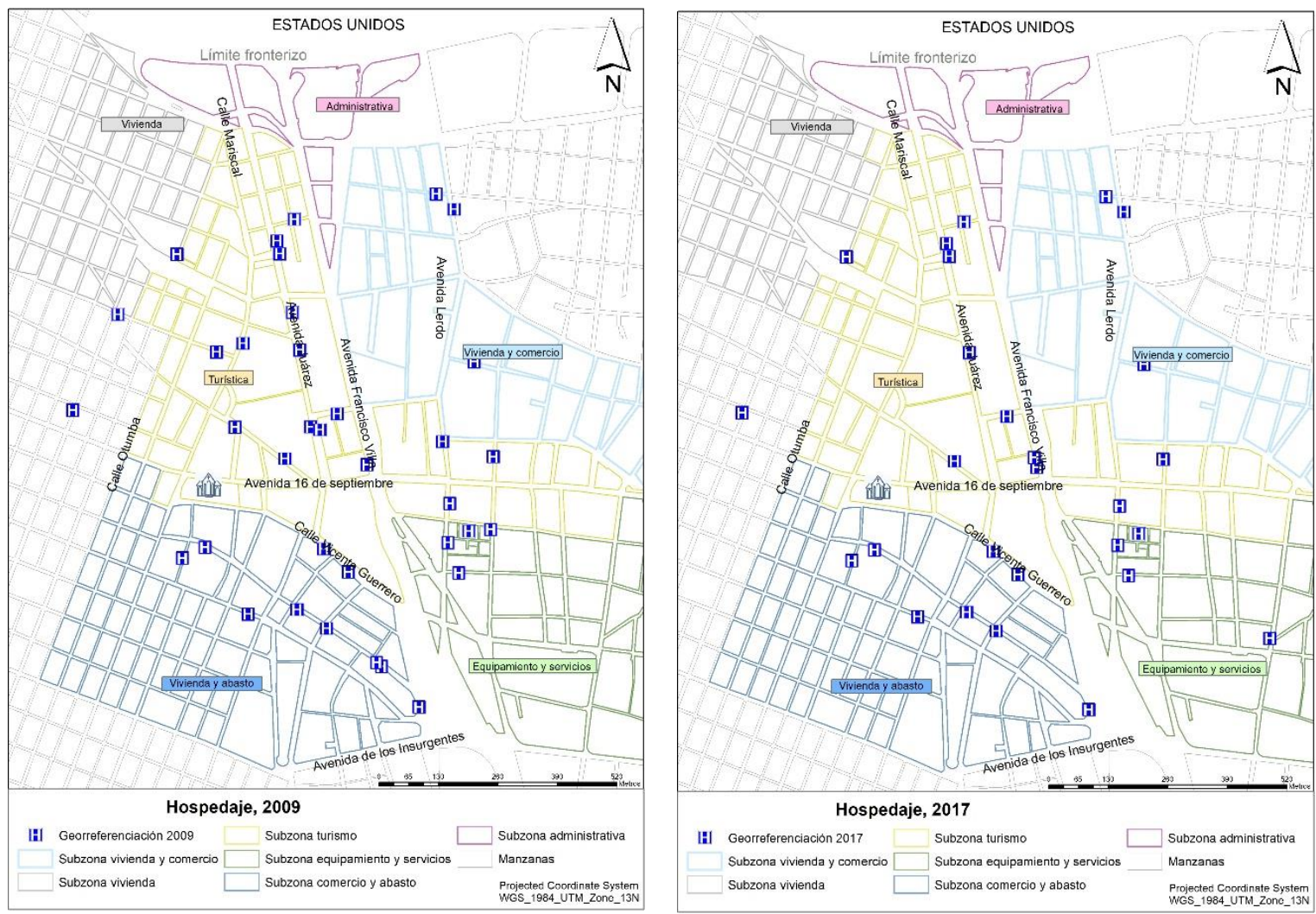

Figura 2. Hoteles y hospedaje 2009-2017.

Fuente: Elaboración propia con base en DENUE y archivo Dirección Comercio Municipal de Ciudad Juárez.

Así mismo, en los recorridos realizados se observó que aquellos comercios que pudieron continuar con sus actividades estaban dirigidos a un perfil de consumidor joven y de mayor poder adquisitivo. En estos comercios han llegado a existir casos de discriminación por cuestiones étnicas, como sucedió con la gobernadora de la colonia Tarahumara, Rosalinda Guadalajara, quien fue impedida de acceder al Club Kentucky por la forma de su vestimenta, siendo complaciente el gobierno municipal con el negocio y justificando a los dueños, ya que solamente se pusieron sellos de suspensión, sin ser totalmente clausurado (Castro, 2016).

El señalamiento de este tipo de actividades comerciales como generadores de violencia y delincuencia en Ciudad Juárez ha permitido que sean los principales afectados al momento de implementarse el PMDUCH. En efecto, es un hecho que el desplazamiento y desaparición de actividades tradicionales del centro de la ciudad han sido paralelos a la renovación urbana, al igual que las personas que habían encontrado un modo de vida en este tipo de negocios. En entrevista con Graciela Espejo, quien es la comisionada del Desarrollo Social y Humano del Centro Histórico (DSHCH), afirmaba que la renovación de esta zona trajo como consecuencia la pérdida de empleos y disminución en la calidad de vida de las trabajadoras sexuales. Se trata, por tanto, de una situación donde el desplazamiento no es únicamente material, sino también simbólico, es decir, que existió otro tipo de desplazamiento menos visible, aplicando estrategias más directas sobre grupos estigmatizados como nocivos para el espacio urbano y que tuvo repercusiones en la población flotante que utilizaba ese espacio, ya sea de visita o de transición.

Ahora bien, haciendo referencia a Janoschka y Sequera (2014) los cambios sufridos en barrios o sectores intervenidos tienden a adoptar nuevas conductas, comportamientos en función al consumo y estilo de vida, que a menudo tiene sus raíces en los sectores de clases 
medias y altas. En otras palabras, el rescate del centro junto con las estrategias implementadas que se suman a la estigmatización de los grupos y actividades comerciales, han tenido como esencia ordenar las calles a través de la homogenización estética, el aumento de cuerpos y sistemas de seguridad $y$, sobre todo, señalando en términos moralizantes las actividades realizadas, a pesar de que estas tienen más de un siglo, como es el comercio sexual alrededor de los hoteles.

Vemos con ello que las políticas de gentrificación, con acento en el desarrollo comercial, se están utilizando para desplazar economías informales y facilitar el surgimiento de procesos de reproducción capitalista, especialmente en el sector inmobiliario formal. Ambas estrategias van de la mano con un discurso basado en la seguridad que justifica la militarización del espacio y la actuación policial para garantizar la eficacia del desplazamiento y desaparición. Es importante señalar que se trata de políticas que tienen por objeto desplazar a los pobres urbanos, de forma directa (demoliendo barrios marginales, embargando o confiscando los terrenos) y de forma indirecta (poniendo en escena el rico patrimonio tangible e intangible para el turismo). En consecuencia, a pesar de contener expresiones diversas, todas estas estrategias tienen en común la presión, la exclusión, el abandono o incluso la represión física para desplazar a la población de estos espacios urbanos específicos, ahora considerados centrales en la rearticulación del capitalismo urbano contemporáneo.

\section{Conclusión}

Este trabajo intenta ser un aporte a las discusiones en torno a los centros históricos latinoamericanos y los procesos de gentrificación comercial. Se trata de un estudio puntual de la zona centro en Ciudad Juárez, Chihuahua; donde se registraron elevados indicadores de violencia e inseguridad a partir de 2007. Como resultado de este fenómeno, se elaboró un discurso que fue utilizado por varias instituciones gubernamentales para justificar intervenciones y renovaciones del perímetro histórico. De esta manera, la discusión se sitúa en el desplazamiento no solo de los grupos sociales o colectivos de esas zonas, sino también en la desaparición de actividades comerciales destinadas a clases económicas bajas, sin respetar su derecho al arraigo o, por lo menos, generando propuestas para su reubicación.

Este estudio es valioso en cuanto al análisis local de cómo las intervenciones urbanas basadas en mejorar el aspecto céntrico de las ciudades tienen como resultado la desaparición o el desplazamiento de actividades comerciales tradicionales como los giros negros, por lo que el trabajo se enfocó en analizar el centro histórico como territorio de un proceso de gentrificación comercial sustentado en estigmatizar este tipo de establecimientos, en especial en la subzona turística.

Se debe resaltar las pocas investigaciones que abordan los efectos de las intervenciones en los centros históricos en función de actividades comerciales estigmatizadas por generar entornos violentos e inseguros. Por tanto, este estudio representa un aporte para siguientes investigaciones que puedan relacionar el fenómeno de la violencia y la inseguridad en términos de actividades económicas, nodos de transporte, intersecciones de calles principales y zonas comerciales nocturnos o espacios fronterizos.

Aunque los informes y estudios han examinado las políticas para regular el expendio de bebida alcohólicas, aún queda pendiente el análisis socioespacial de los entornos locales y sus repercusiones en las dinámicas sociales, las cuales han sido modificadas por las intervenciones urbanas en este tipo de contextos de violencia.

Los hallazgos indican la desaparición de estas actividades donde sus consumidores eran de clases sociales bajas, sobreviviendo aquellos negocios dirigidos a sectores jóvenes y de alto consumo. Lo anterior contraviene la estrategia y objetivos de los dos programas de intervención Todos Somos Juárez y $\mathrm{PMDUCH}$, enfocados en impulsar una cohesión social y disminuir los indicadores de violencia. Así, pues, el fin superior no explícito en estos programas era consolidar la zona centro como un nodo turístico fronterizo.

En consecuencia, se observa una transformación drástica del perímetro histórico de Ciudad Juárez debido a la gentrificación, cambiando no el uso del suelo sino el comportamiento del consumidor y clase social al que está dirigido. Por ello, era necesario justificar la intervención urbana basada en el fenómeno transversal de violencia que se vivió a partir de 2007 y que repercutió en la 
generación de las políticas que tuvieron como esencia desplazar a las clases sociales marginales por medio de diversas estrategias, ya sean policiacas o de negociación con los dueños de predios.

Si bien en este escenario se moderó el uso del suelo como un instrumento de política urbana para disminuir el fenómeno de violencia, no deja de ser relevante que existieran otros factores como las desventajas socioeconómicas de los habitantes (el elemento más fuerte y consistente para la mayoría de los estudios). Así mismo, se tiene una política de desplazamiento no solamente de colectivos y estratos económicos bajos, sino también de actividades económicas destinadas para ellos.

\section{Referencias}

August, M. (2014). Challenging the rhetoric of stigmatization: The benefits of concentrated poverty in Toronto's Regent Park. Environment and Planning A, 46(6), 1317-1333. https://doi.org/10.1068/a45635

Carrión, F. (2005). El centro histórico como proyecto y objeto de deseo. EURE (Santiago), 31(93), 89-100. http://dx.doi.org/10.4067/S025071612005009300006

Carrión, F. (2017). Centralidades históricas: desafíos de una realidad en permanente construcción. Seminario permanente 2017: La ciudad posible. Conferencia llevada a cabo en el Instituto Municipal de Investigación y Planeación (IMIP), Ciudad Juárez, México.

Carrión, F. (19 mayo de 2014). Urbicidio, centros históricos y la ciudad. Recuperado de www.youtube.com/watch?v=QGPijZ87pZE

Castilhos, R. (2015). Researching the post-industrial city: Assessing the relations between space, markets, and society in urban places. Recuperado de http://www.acrwebsite.org/volumes/v43/acr vol43 1019841.pdf

Castro, S. (2016). Colocan sellos de suspensión a bar Kentucky. Recuperado de http://diario.mx/Local/2016
Si se reflexiona de manera profunda se puede observar el desplazamiento como elemento notable para la extracción y acumulación capitalista en los procesos urbanos actuales, transversal a los procesos de gentrificación. Por esta razón es necesario impulsar estrategias que sean incluyentes, respetando en la medida posible el derecho al arraigo, o bien, entregando propuestas de relocalización de estas actividades sin que sean estigmatizados sus consumidores. A su vez, los planeadores y autoridades debieran tener presente las características microlocales de los barrios y colonias, incluyendo el uso del suelo, para tomar decisiones acertadas en relación con un comercio responsable con su entorno [B]

Castañón, A. y Carmona, B. (2015). Encierra Hotel Verde historias de pesadilla. Recuperado de http://diario.mx/micrositios/hotel-verde-en-la-zonacentro-de-juarez-escenario-de-la-explotacion/

Chabat, J. (2010). La respuesta del gobierno de Calderón al desafío del narcotráfico: entre lo malo y lo peor. Documentos de trabajo del CIDE. División de Estudios Internacionales, 196, 1-18. Recuperado de http://www.libreriacide.com/librospdf/DTEI-196.pdf

Chaparro, L. (2013). Gobierno derrumba la historia de Ciudad Juárez. Recuperado de http://www.sinembargo.mx/08-04-2013/578404

Ciccolella, P. (2010). Metrópolis y desarrollo urbano más allá de la globalización. Hacia una geografía crítica de la ciudad latinoamericana. Scripta Nova. Revista Electrónica de Geografía y Ciencias Sociales, 14. http://revistes.ub.edu/index.php/ScriptaNova/article /view/1653/1629

Contreras, E. (2017). Renovación y desplazamientos urbanos. Buenos Aires: Investigaciones latinoamericanas. Recuperado de http://surbanistas.org/wpcontent/uploads/2017/01/Renovacio\%CC\%81n-yDesplazam-Urb EdgardoContrerasNossa web.pdf 
Cordero Gómez del Campo, L. y Salinas Arreortua, L. (2017). Gentrificación comercial. Espacios escenificados y el modelo de los mercados gourmet. Revista de Urbanismo, 37, 1-12. https://doi.org/10.5354/0717-5051.2017.45735

Delgadillo, V. (2015). Desafíos para el estudio de desplazamientos sociales en los procesos de gentrificación. Recuperado de http://contestedcities.net/working-papers/wpcontent/uploads/sites/8/2015/01/WPCC-15002DelgadilloVictor DesafiosEstudioDesplazamiento.pdf

Delgadillo, V. (2016). Ciudad de México, quince años de desarrollo urbano intensivo: la gentrificación percibida. Revista INVI, 31(88), 101-129. http://dx.doi.org/10.4067/S071883582016000300004

Espejo, G. (20 de junio de 2017). Entrevista de los cambios del centro histórico de Ciudad Juárez por la renovación.

Ferm, J. (2016) Preventing the displacement of small businesses through commercial gentrification: Are affordable workspace policies the solution? Planning Practice \& Research, 31(4), 402-419, https://doi.org/10.1080/02697459.2016.1198546

Gastaldi, F. y Camerin, F. (2015). El proceso de gentrificación en Génova a partir de las trasformaciones urbanas de 1992. Revista de Urbanismo, 33, 132-154. https://doi.org/10.5354/0717-5051.2015.36549

González, M. (2010). Coche bomba mata a 3 en Juárez. Recuperado de http://archivo.eluniversal.com.mx/

Gottdiener, M., Hutchison, R., \& Ryan, M. T. (2014). The new urban sociology. Hachette UK.

Gobierno Federal. (2010). Todos Somos Juárez, Reconstruyamos la Ciudad. Ciudad Juárez: Gobierno Federal. Recuperado de http://www.conadic.salud.gob.mx/pdfs/todos somos juarez 28junio.pdf

Institute for Economics and Peace. (2016). México Peace Index. México: IEP. Recuperado de http://economicsandpeace.org/
Instituto Municipal de Investigación y Planeación, IMIP. (2013). Plan Maestro de Desarrollo Urbano del Centro Histórico de Ciudad Juárez. Ciudad Juárez: Gobierno Municipal de Ciudad Juárez.

Inzulza-Contardo, J. y Díaz Parra, I. (2016). Desastres naturales, destrucción creativa y gentrificación: estudio de casos comparados en Sevilla (España), Ciudad de México (México) y Talca (Chile). Revista de Geografía Norte Grande, 64, 109-128. http://dx.doi.org/10.4067/S071834022016000200008

Jamieson, P. (1993). A survey history of Fort Bliss 18901940. Army Air Defense Artillery Center Fort Bliss. Recuperado de http://www.dtic.mil/cgibin/GetTRDoc?AD=ADA277986\&Location $=U 2 \&$ doc $=\mathrm{G}$ etTRDoc.pdf

Janoschka, M. y Sequera, J. (2014). Procesos de gentrificación y desplazamiento en América Latina, una perspectiva comparativista. Recuperado de http://contested-cities.net/wpcontent/uploads/2014/07/2014CC Janoschka Seque ra Desplazamiento AL.pdf

Lara, P y Enrique, J. (2011). La guerra contra el narcotráfico: ¿una guerra perdida? Espacios públicos, 14(30). Recuperado de http://www.redalyc.org/html/676/67618934014/

Lefebvre, H. (1974). La producción del espacio. Madrid: Gracel Asociados.

López-Morales, E., Klet, I. G., y Corvalán, D. M. (2014). Captura desigual de renta de suelo y desplazamiento exclusionario. Indicadores generales del proceso de gentrificación en Santiago de Chile, 2000-2012. Cadernos Metrópole, 16(32), 565-586. http://dx.doi.org/10.1590/2236-9996.2014-3212

Organización de las Naciones Unidas para la Educación, la Ciencia y la Cultura, Unesco. (2013). Nueva vida para las ciudades históricas. El planteamiento de los paisajes urbanos históricos. Recuperado de https://whc.unesco.org/document/128593.

Padilla, H. A. P. D., Andrade, C. A. O., y Salas, L. A. (2014). Ciudad Juárez y la necesidad de política: de la ciudad real a la ideal. Universidad Autónoma de Ciudad Juárez. 
Peña, L. y Sandoval, L. (2010). Historia del cabaret y vida nocturna como transformadores de la identidad cultural en el centro de Ciudad Juárez, Chihuahua, México. Barranquilla, Colombia: XXXIII Encuentro RNIU.

Plan Estratégico de Juárez. (2016). Informe 2016. Así estamos Juárez. Sistemas de indicadores de calidad de vida. Ciudad Juárez: Autor.

Pradilla, E. (2013). La ciudad capitalista en el patrón neoliberal de acumulación en América Latina. Seminário Internacional A cidade Neoliberal na América Latina. http://dx.doi.org/10.1590/22369996.2014-3102

Rubio, J. (2013). La otra cara del trabajo sexual en Ciudad Juárez. Recuperado de http://borderzine.com/2013/03/la-otra-cara-deltrabajo-sexual-en-ciudad-juarez/

Sabatini, F., Rasse, A., Cáceres, G., Robles, M. S., y Trebilcock, M. P. (2017). Promotores inmobiliarios, gentrificación y segregación residencial en Santiago de Chile. Revista Mexicana de Sociología, 79(2), 229-260. Recuperado de http://www.scielo.org.mx/scielo.php?script=sci artte $\underline{x t \& p i d=S 0188-25032017000200229 \& \operatorname{lng}=e s \& t \operatorname{lng}=e s}$.
Smith, N. (1979). Toward a theory of gentrification a back to the city movement by capital, not people. Journal of the American Planning Association, 45(4), 538-548. https://doi.org/10.1080/01944367908977002

Vázquez Moyers, A. y Espino Sánchez, G. (2015). La producción discursiva en la guerra contra el narcotráfico en el sexenio de Calderón: en busca de la legitimidad perdida. Discurso \& Sociedad, 4, 492-518.

Wacquant, L. (2006). Castigar a los parias urbanos. Antípoda. Revista de Antropología y Arqueología, 2, 59-66.

http://www.scielo.org.co/scielo.php?script=sci arttex t\&pid=S1900-54072006000100005\&lng=en\&tlng=pt

Wacquant, L., Slater, T., y Borges Pereira, V. (2014). Estigmatización territorial en acción. Revista INVI, 29(82), 219-240. https://dx.doi.org/10.4067/S071883582014000300008

Wolf, S. y Morayta, G. C. (2011). La guerra de México contra el narcotráfico y la Iniciativa Mérida: piedras angulares en la búsqueda de legitimidad. Foro Internacional, 669-714. http://www.redalyc.org/html/599/59923588003/ 